\title{
ON THE STABILITY FOR LINEAR VISCOELASTIC SOLIDS
}

\author{
BY \\ C. GIORGI (Dipartimento di Elettronica per l'Automazione, I-25123 Brescia, Italy) \\ AND \\ B. LAZZARI (Dipartimento di Matematica, 40127 Bologna, Italy)
}

\begin{abstract}
The stability and asymptotic behaviour of solutions to the dynamical initial past-history problem within the theory of linear viscoelasticity is investigated. First, a family of free energies is introduced as a Liapunov functional via the energy method. Then, emphasizing the role of thermodynamic inequalities, the asymptotic stability property is derived by means of some original a priori integral estimates of the solution.
\end{abstract}

1. Introduction. We shall consider a viscoelastic solid $\mathcal{B}$ occupying a bounded region $\Omega \subset \mathbb{R}^{3}$ with smooth boundary $\partial \Omega$ in an unstressed reference placement. Let $\mathbf{x} \in \Omega$ be the position vector of any point of $\mathcal{B}$. The motion of the body is given by the displacement vector $\mathbf{u}(\mathbf{x}, t)$ as a function of position $\mathbf{x}$ and time $t$. As usual, in linearized theories the deformation is described by the infinitesimal strain tensor $\mathbf{E}=\operatorname{sym} \nabla \mathbf{u}$.

The theory of linear isothermal viscoelasticity [1]-[4] is characterized by a stress-strain relation of the form

$$
\mathbf{T}(\mathbf{x}, t)=\boldsymbol{T}\left(\mathbf{E}^{t}(\mathbf{x}, \cdot)\right), \quad(\mathbf{x}, t) \in \Omega \times \mathbb{R}^{+},
$$

where $\mathbf{T}$ is the stress tensor, $\mathbf{E}^{t}$ is the strain tensor history up to time $t$, i.e., $\mathbf{E}^{t}(\mathbf{x}, s)=$ $\mathbf{E}(\mathbf{x}, t-s), s \in \mathbb{R}^{+}$, and

$$
\boldsymbol{T}\left(\mathbf{E}^{t}(\mathbf{x}, \cdot)\right)=\mathbf{G}_{0}(\mathbf{x}) \mathbf{E}^{t}(\mathbf{x}, 0)+\int_{0}^{\infty} \mathbf{G}^{\prime}(\mathbf{x}, s) \mathbf{E}^{t}(\mathbf{x}, s) d s .
$$

Here, both $\mathbf{G}_{0}$ and $\mathbf{G}^{\prime}$ are fourth-order tensor-valued functions called instantaneous elastic modulus and Boltzmann function, respectively. Following the viewpoint underlying previous results on linear viscoelasticity [4], the minimal set of properties for modelling a viscoelastic solid is considered. First, we assume

$$
\mathbf{G}^{\prime} \in L^{1}\left(\mathbb{R}^{+}, L^{\infty}(\Omega)\right) .
$$

Received November 1, 1994.

1991 Mathematics Subject Classification. Primary 73F15, 73H99, 73B30, 45K05. 
As a consequence, the relaxation function $\mathbf{G}(\mathbf{x}, s)=\mathbf{G}_{0}(\mathbf{x})+\int_{0}^{s} \mathbf{G}^{\prime}(\mathbf{x}, \tau) d \tau$ is well defined along with the limit $\mathbf{G}_{\infty}(\mathbf{x})=\lim _{s \rightarrow \infty} \mathbf{G}(\mathbf{x}, s)$.

For the sake of simplicity, the Boltzmann function $\mathbf{G}^{\prime}$ is taken to be symmetric. ${ }^{1}$ In this regard, as is well known [5], we observe that thermodynamics implies the symmetry of $\mathbf{G}_{0}$ and $\mathbf{G}_{\infty}$, not the symmetry of the relaxation function $\mathbf{G}$. On the other hand, as proven in [6], the Second Law of Thermodynamics gives no condition on the definiteness of $\mathbf{G}^{\prime}$ but requires that $\mathbf{G}_{s}^{\prime}$, the half-range Fourier-sine transform of the Boltzmann function, be negative definite, ${ }^{2}$ namely

$$
\mathbf{G}_{s}^{\prime}(\mathbf{x}, \omega)<\mathbf{0} \text { a.e. in } \Omega \text { for any } \omega \in \mathbb{R}^{++} .
$$

In addition, the solid character of the body is prescribed by requiring that the equilibrium elastic modulus $\mathbf{G}_{\infty}(\mathbf{x})$ is positive definite in $\Omega$.

All previous assumptions on the relaxation function may be summarized as follows:

$\mathbb{P}_{1}$. G is a symmetric fourth-order tensor-valued function continuous in $\bar{\Omega} \times \mathbb{R}^{+}$and differentiable in $\Omega \times \mathbb{R}^{+}$.

$\mathbb{P}_{2}$. $\mathbf{G}^{\prime}$ satisfies a "weak" fading memory principle, namely (1.2).

$\mathbb{P}_{3} . \mathbf{G}_{\infty}$ is positive definite, accounting for the solid character of the body.

$\mathbb{P}_{4}$. $\mathbf{G}^{\prime}$ satisfies a thermodynamic restriction, namely (1.3).

$\mathbb{P}_{5}$. The limit of $\mathbf{G}^{\prime}(\mathbf{x}, s)$ as $s \rightarrow 0^{+}$exists and is bounded a.e. in $\Omega$.

Henceforth, whenever no ambiguity arises, the dependence on position $\mathbf{x}$ will be understood and not written. Moreover, the Fourier transform of any smooth function $f$ on $\mathbb{R}$ will be denoted by $\hat{f}(\omega)$. Regarding all functions defined on $\mathbb{R}^{+}$as functions on $\mathbb{R}$ that vanish identically on $\mathbb{R}^{-}$, we set $\hat{f}=f_{c}-i f_{s}$, where $f_{c}$ and $f_{s}$ are the half-range Fouriercosine and Fourier-sine transforms, respectively. Remarkably, by the Fourier inversion formula we have

$$
\mathbf{G}(s)-\mathbf{G}_{0}=\frac{2}{\pi} \int_{0}^{\infty} \frac{1-\cos \omega s}{\omega} \mathbf{G}_{s}^{\prime}(\omega) d \omega .
$$

Thereby, the limit of (1.4) times $s^{-1}$ as $s \rightarrow 0^{+}$is just the initial time derivative $\mathbf{G}^{\prime}\left(0^{+}\right)$, which exists and is negative semidefinite because of $\mathbb{P}_{4}$ and $\mathbb{P}_{5}$.

Incidentally, taking the limit as $s \rightarrow \infty$, from (1.4) it follows that

$$
\mathbf{G}_{\infty}-\mathbf{G}_{0}=\frac{2}{\pi} \int_{0}^{\infty} \frac{1}{\omega} \mathbf{G}_{s}^{\prime}(\omega) d \omega,
$$

which yields the positive definiteness of $\mathbf{G}_{0}$ because of $\mathbb{P}_{3}$ and $\mathbb{P}_{4}$. By $\mathbb{P}_{1}-\mathbb{P}_{5}$ and the boundedness of the right-hand side of $(1.5), \mathbf{G}_{s}^{\prime}$ is continuous and

$$
\mathbf{G}_{s}^{\prime} \text { and } \frac{1}{\omega} \mathbf{G}_{s}^{\prime} \text { belong to } L^{1}\left(\mathbb{R}^{+}, L^{\infty}(\Omega)\right) \text {. }
$$

When viscoelastic material behaviour is framed within the theory of materials with fading memory, an appropriate normed function space is required. Of course, a norm is basic in dealing with differential calculus on memory functionals and, specifically, in scrutinizing stability. Usually, the norm is defined in terms of an influence function $h$

\footnotetext{
${ }^{1} \mathrm{~A}$ fourth-order tensor $\mathbf{F}$ is said to be symmetric if $\mathbf{A} \cdot \mathbf{F B}=\mathbf{B} \cdot \mathbf{F A} \forall \mathbf{A}, \mathbf{B} \in \mathrm{Sym}$.

${ }^{2}$ A symmetric fourth-order tensor $\mathbf{F}$ is said to be positive (negative) definite or semidefinite if $\mathbf{A} \cdot \mathbf{F A}>$ 0 or $\geq 0(<0$ or $\leq 0) \forall \mathbf{A} \in \operatorname{Sym} \backslash\{\mathbf{0}\}$. For ease in writing we shall denote $\mathbf{F}>\mathbf{0}$ or $\mathbf{F} \geq \mathbf{0}(<\mathbf{0}$ or $\leq \mathbf{0})$.
} 
which models the fading memory property but is not strictly related to the material constitutive features. For instance, we may consider the customary norm

$$
\left\|\mathbf{E}^{t}\right\|_{h}^{2}=|\mathbf{E}(t)|^{2}+\int_{0}^{\infty}\left|\mathbf{E}^{t}(s)\right|^{2} h(s) d s,
$$

involving a positive monotone decreasing influence function $h \in L^{1}\left(\mathbb{R}^{+}\right)$such that

$$
\int_{0}^{\infty}\left|\mathbf{G}^{\prime}(s)\right|^{2} h^{-1}(s) d s<+\infty .
$$

General properties such as thermodynamical principles and stability statements, however, should not involve any norm that strongly depends on a subjective choice of an influence function. In addition, it is well known that thermodynamic potentials (e.g., free energy) are hard to construct and, generally, are not continuous relative to fading memory norms like (1.7). This is a serious lack, since the continuity of such functionals is crucial in connection with Liapunov-type stability.

In order to avoid these drawbacks, some authors [7] constructed history norms through positive-definite quadratic forms that are free from arbitrary influence functions. Actually, inequality (1.3) and property (1.6) allow them to define history spaces where $\mathbf{G}_{s}^{\prime}$ enters the definition of the norm as an intrinsic influence function that naturally accounts for the memory properties of the material. More importantly, a continuous quadratic functional endowed with the characteristic properties of the free energy corresponds to each norm. Following this viewpoint, free energies were employed by Fabrizio [8] as Lyapunov functionals in scrutinizing stability, which is called the energy method.

This paper deals with solutions to the following dynamical initial past-history problem with Dirichlet boundary conditions:

$$
\left\{\begin{array}{l}
\frac{\partial^{2}}{\partial t^{2}} \mathbf{u}(\mathbf{x}, t)-\nabla \cdot \mathbf{T}(\mathbf{x}, t)=\mathbf{f}(\mathbf{x}, t), \quad(\mathbf{x}, t) \in \Omega \times(0, \tau), \\
\mathbf{T}(\mathbf{x}, t)=\mathbf{G}_{0}(\mathbf{x}) \nabla \mathbf{u}^{t}(\mathbf{x}, 0)+\int_{0}^{\infty} \mathbf{G}^{\prime}(\mathbf{x}, s) \nabla \mathbf{u}^{t}(\mathbf{x}, s) d s, \quad(\mathbf{x}, t) \in \Omega \times(0, \tau), \\
\mathbf{u}(\mathbf{x}, t)=\mathbf{g}(\mathbf{x}, t), \quad(\mathbf{x}, t) \in \partial \Omega \times(0, \tau), \\
\mathbf{u}^{t}(\mathbf{x}, s)=\mathbf{u}^{0}(\mathbf{x}, s-t), \quad(\mathbf{x}, t) \in \Omega \times(0, \tau), s \geq t,
\end{array}\right.
$$

where $\mathbf{u}$ is the displacement vector and $\mathbf{u}^{t}$ is the displacement history up to time $t$, i.e., $\mathbf{u}^{t}(\mathbf{x}, s)=\mathbf{u}(\mathbf{x}, t-s), s \in \mathbb{R}^{+}$. The body force $\mathbf{f}$, the displacement boundary value $\mathbf{g}$, and the displacement initial past-history $\mathbf{u}^{0}$ are given, of course.

As is well known (see, e.g., [9]), existence, uniqueness, and continuous dependence of solutions to (1.8) easily follow from the positive definiteness of $\mathbf{G}_{0}$ alone. In spite of this, notable results about asymptotic stability have been obtained by Dafermos [10] at the cost of strong additional assumptions involving $\mathbf{G}^{\prime}$ and $\mathbf{G}^{\prime \prime}$.

From a different viewpoint, a general result on asymptotic behaviour was established by Fabrizio and Lazzari [11]. Their approach heavily rests on the thermodynamic property $\mathbb{P}_{4}$ but requires rather mild assumptions on $\mathbf{G}$. Our aim, here, is to perform a general stability analysis of linear viscoelastic processes on the basis of $\mathbb{P}_{1}-\mathbb{P}_{5}$, thus emphasizing the role of thermodynamic inequalities such as (1.3). 
Although our original contributions mainly refer to the second part of this paper (sections 3, 4, and 5), for the sake of completeness, the first part (section 2) outlines the formerly quoted results on thermodynamic potentials $[7,8]$ and criticizes their application to the energy method. In this connection, we point out that stability relative to any viscoelastic energy norm implies stability relative to the usual elastic one.

In the second part we look for suitable a priori integral estimates involving a specific "weighted" norm of the solution. These estimates, along with asymptotic properties proven in [11], allow us to improve previous stability results. Emphasizing the connection between thermodynamics and asymptotic behaviour of solutions, the asymptotic stability property for linear viscoelastic solids is achieved on the basis of the thermodynamic restriction $\mathbb{P}_{4}$. It is worth noting that asymptotic stability (but not simple stability) fails if $\mathbb{P}_{4}$ is weakened, in particular, when the sign of inequality (1.3) is not strict. This is proven by a simple counterexample. Actually, if there exists a circular frequency $\omega^{*}>0$ such that $\mathbf{G}_{s}^{\prime}\left(\omega^{*}\right)$ is positive semidefinite, but not definite, then a special sinusoidal-intime past-history perturbation can support an oscillating solution of the same frequency $\omega^{*}$. Let us also point out that $\mathbb{P}_{4}$ also provides a necessary and sufficient condition to achieve the inversion of the constitutive functional (1.1) in the space $L^{1}$ (or $L^{\infty}$ ).

2. Free energies and stability. In the sequel, via the energy method, stability results are established involving a specific family of continuous free energy functionals defined on the basis of [7].

Definition 1. A set $\Phi$ of strain histories $\mathbf{E}^{t}: \mathbb{R}^{+} \rightarrow$ Sym is said to be admissible if the followirng properties hold:

i) $\mathbf{E}^{t}$ is Fourier transformable (in a distributional sense);

ii) $\int_{0}^{\infty} \mathbf{G}^{\prime}(s) \mathbf{E}^{t}(s) d s<\infty$;

iii) if $\mathbf{E}^{t} \in \Phi$ then each strain-history $\mathbf{E}^{t+\tau}, \tau>0$, such that $\mathbf{E}^{t+\tau}(s+\tau)=\mathbf{E}^{t}(s)$, $s \in \mathbb{R}^{+}$, is an element of $\Phi$.

Let ${ }_{r} \Phi$ denote the admissible set of all past-histories ${ }_{r} \mathbf{E}^{t}$ that are obtained from histories $\mathbf{E}^{t} \in \Phi$ by restriction to $\mathbb{R}^{++}$. As a consequence, we have

$$
\Phi=\operatorname{Sym} \times{ }_{r} \Phi, \quad \mathbf{E}^{t}=\left(\mathbf{E}^{t}(0),{ }_{r} \mathbf{E}^{t}\right) .
$$

Henceforth, we denote by $\Phi_{\mathbf{E}}$ the subset of all admissible histories with present value $\mathbf{E}$, i.e., $\mathbf{E}^{t}(0)=\mathbf{E}$, and by $\mathbf{E}^{\dagger}$ the constant history taking the value $\mathbf{E}$, viz., $\mathbf{E}^{\dagger}(s)=\mathbf{E}$, $s \in \mathbb{R}^{+}$.

DEFinition 2. A free energy, relative to a given constitutive equation (1.1), is a functional $\Psi: \Phi \rightarrow \mathbb{R}$ endowed with the following properties:

i) $\Psi$ is continuous and differentiable with respect to the first argument so that

$$
\boldsymbol{T}\left(\mathbf{E}^{t}\right)=\frac{\partial}{\partial \mathbf{E}} \Psi\left(\mathbf{E}^{t}(0),{ }_{r} \mathbf{E}^{t}\right)
$$

ii) $\Psi$ is differentiable with respect to time and satisfies the inequality

$$
\frac{\partial}{\partial s} \Psi\left(\mathbf{E}^{t+s}\right) \leq \boldsymbol{T}\left(\mathbf{E}^{t+s}\right) \cdot \frac{\partial}{\partial s} \mathbf{E}(t+s)
$$

for all $\mathbf{E}^{t} \in \Phi$ and $s \geq 0$ such that $\frac{\partial}{\partial s} \mathbf{E}(t+s)$ is continuous; 
iii) $\Psi$ is minimal on constant histories; namely, for each $\mathbf{E} \in$ Sym,

$$
\Psi\left(\mathbf{E}^{t}\right) \geq \Psi\left(\mathbf{E}^{\dagger}\right), \quad \forall \mathbf{E}^{t} \in \Phi_{\mathbf{E}} .
$$

where the equality holds if and only if $\mathbf{E}^{t}$ is the constant history $\mathbf{E}^{\dagger}$.

In [7] the main result about free energy functionals involves a positive-definite, fourthorder tensor-valued function $\mathbf{N}$ defined on $\mathbb{R}^{+}$such that:

$\mathbb{A}_{1}$. the Fourier transforms of $\mathbf{N}$ and $\mathbf{G}^{\prime} \mathbf{N}^{-1}$ exist (in a distributional sense) and, moreover, $\mathbf{N}_{s}$ and $\mathbf{K}=-\left(\mathbf{G}^{\prime} \mathbf{N}^{-1}\right)_{s}$ are positive definite on $\mathbb{R}^{++}$;

$\mathbb{A}_{2}$. for any strain-history $\mathbf{E}^{t} \in \Phi$, both $\mathbf{N}$ and $\mathbf{N}^{\prime}=\frac{d}{d t} \mathbf{N}$ obey the inequality

$$
\int_{0}^{\infty}\left\{\left(\mathbf{N}^{\prime} \mathbf{E}^{t}\right)_{s} \cdot \mathbf{J}\left[\left(\mathbf{N} \mathbf{E}^{t}\right)_{s}-\mathbf{N}_{s} \mathbf{E}(t)\right]+\left[\left(\mathbf{N}^{\prime} \mathbf{E}^{t}\right)_{c}-\mathbf{N}_{c}^{\prime} \mathbf{E}(t)\right] \cdot \mathbf{J}\left(\mathbf{N} \mathbf{E}^{t}\right)_{c}\right\}(\omega) d \omega \leq 0
$$

where $\mathbf{J}=\mathbf{K}^{1 / 2} \mathbf{N}_{s}^{-1} \mathbf{K}^{1 / 2}$ is positive definite on $\mathbb{R}^{++}$because of $\mathbb{A}_{1}$.

TheOREM $1([7, \S 4])$. Let $\mathbf{N}$ be a positive definite tensor satisfying $\mathbb{A}_{1}-\mathbb{A}_{2}$. Then

$$
\begin{aligned}
& \Psi_{\mathbf{N}}\left(\mathbf{E}(t),{ }_{r} \mathbf{E}^{t}\right)=\frac{1}{2} \mathbf{E}(t) \cdot \mathbf{G}_{\infty} \mathbf{E}(t) \\
& \quad+\frac{1}{\pi} \int_{0}^{\infty}\left\{\left[\left(\mathbf{N E}^{t}\right)_{s}-\mathbf{N}_{s} \mathbf{E}(t)\right] \cdot \mathbf{J}\left[\left(\mathbf{N E}^{t}\right)_{s}-\mathbf{N}_{s} \mathbf{E}(t)\right]+\left(\mathbf{N E}^{t}\right)_{c} \cdot \mathbf{J}\left(\mathbf{N E}^{t}\right)_{c}\right\}(\omega) d \omega
\end{aligned}
$$

is a free energy functional (in the sense of Definition 2) on $\Phi_{\mathbf{N}}$, where

$$
\Phi_{\mathbf{N}}=\left\{\mathbf{E}^{t}: \mathbf{N E}^{t} \in L^{2}\left(\mathbb{R}^{+}\right) \text {and } \Psi_{\mathbf{N}}\left(\mathbf{E}(t),{ }_{r} \mathbf{E}^{t}\right)<\infty\right\} .
$$

If $\mathbf{G}$ satisfies $\mathbb{P}_{1}-\mathbb{P}_{5}$ then each $\Psi_{\mathbf{N}}$ defines a norm, namely,

$$
\left\|\mathbf{E}^{t}\right\|_{\mathbf{N}}^{2}=\Psi_{\mathbf{N}}\left(\mathbf{E}(t),{ }_{r} \mathbf{E}^{t}\right)
$$

and each space $\mathcal{H}_{\mathbf{N}}$, obtained as the completion of $\Phi_{\mathbf{N}}$ relative to this norm, is a Banach space. Trivially, $\Psi_{\mathbf{N}}$ is well defined and turns out to be continuous on $\mathcal{H}_{\mathbf{N}}$.

REMARK 1. Let $\mathbf{G}$ be any function satisfying $\mathbb{P}_{1}-\mathbb{P}_{5}$. The choice $\mathbf{N}=\mathbf{1}$ complies with $\mathbb{A}_{1}-\mathbb{A}_{2}$ and the corresponding free energy, $\Psi_{1}$, is given by

$$
\begin{aligned}
\Psi_{\mathbf{1}}\left(\mathbf{E}(t),{ }_{r} \mathbf{E}^{t}\right)= & \frac{1}{2} \mathbf{E}(t) \cdot \mathbf{G}_{\infty} \mathbf{E}(t) \\
& \quad-\frac{1}{\pi} \int_{0}^{\infty}\left\{\left[\omega \mathbf{E}_{s}^{t}-\mathbf{E}(t)\right] \cdot \frac{1}{\omega} \mathbf{G}_{s}^{\prime}\left[\omega \mathbf{E}_{s}^{t}-\mathbf{E}(t)\right]+\mathbf{E}_{c}^{t} \cdot \frac{1}{\omega} \mathbf{G}_{s}^{\prime} \mathbf{E}_{c}^{t}\right\}(\omega) d \omega .
\end{aligned}
$$

Moreover, $\Psi_{1}$ is the maximal free energy; namely, for any $\mathbf{N}$ satisfying $\mathbb{A}_{1}-\mathbb{A}_{2}$ we have

$$
\Psi_{\mathbf{N}}\left(\mathbf{E}(t),{ }_{r} \mathbf{E}^{t}\right) \leq \Psi_{\mathbf{1}}\left(\mathbf{E}(t),{ }_{r} \mathbf{E}^{t}\right) \quad \forall \mathbf{E}^{t} \in \mathcal{H}_{\mathbf{1}} \subset \mathcal{H}_{\mathbf{N}}
$$

REMARK 2. All constant histories belong to the Banach space $\mathcal{H}_{1}$; in fact,

$$
\left\|\mathbf{E}^{\dagger}\right\|_{1}^{2}=\frac{1}{2} \mathbf{E} \cdot \mathbf{G}_{\infty} \mathbf{E}
$$

Nevertheless, $\mathcal{H}_{1}$ cannot contain any oscillating histories, because its norm does not exhibit the customary "fading memory" property. 
REMARK 3. Let $\mathbf{G}$ be any function satisfying $\mathbb{P}_{1}-\mathbb{P}_{5}$ and such that $\mathbf{G}^{\prime}<0$ and $\mathbf{G}^{\prime \prime} \geq 0$. Then, assuming $\mathbf{N}=\left(-\mathbf{G}_{s}^{\prime}\right)^{1 / 2}$, we have $\mathbf{J}=\mathbf{1}$ and the functional (2.4) takes the form of the so-called "Graffi-Volterra" free energy, namely,

$$
\Psi_{\mathbf{G}}\left(\mathbf{E}(t),{ }_{r} \mathbf{E}^{t}\right)=\frac{1}{2} \mathbf{E}(t) \cdot \mathbf{G}_{\infty} \mathbf{E}(t)-\frac{1}{2} \int_{0}^{\infty} \mathbf{G}^{\prime}(s)\left[\mathbf{E}^{t}(s)-\mathbf{E}(t)\right] \cdot\left[\mathbf{E}^{t}(s)-\mathbf{E}(t)\right] d s .
$$

The corresponding norm $\|\cdot\|_{\mathbf{G}}$, given by (2.5), is naturally endowed with the fading memory property and then all bounded histories belong to $\mathcal{H}_{\mathbf{G}}$.

By linearity, the stability problem for the evolutive system (1.8) reads as follows: to determine the set $\mathcal{U}$ of initial past-history perturbations $\mathbf{u}^{0}$ such that the zero-history solution (namely $\mathbf{u}^{\tau}=\mathbf{0}^{\dagger}$ ) to the homogeneous system

$$
\left\{\begin{array}{l}
\frac{\partial^{2}}{\partial t^{2}} \mathbf{u}^{t}(0)-\nabla \cdot \mathbf{T}(t)=0, \quad \text { on } \Omega \times(0, \tau), \\
\mathbf{T}(t)=\mathbf{G}_{0} \nabla \mathbf{u}^{t}(0)+\int_{0}^{\infty} \mathbf{G}^{\prime}(s) \nabla \mathbf{u}^{t}(s) d s, \quad \text { on } \Omega \times(0, \tau), \\
\mathbf{u}^{t}(0)=\mathbf{0}, \quad \text { on } \partial \Omega \times(0, \tau), \\
\mathbf{u}^{t}(s)=\mathbf{u}^{0}(s-t), \quad \text { on } \Omega \times(0, \tau), s \geq t,
\end{array}\right.
$$

is stable (in the sense of Lyapunov) relative to a fixed "energy norm".

A straightforward calculation yields the classical theorem of expended power ${ }^{3}$

$$
\frac{1}{2}\left\|\frac{\partial}{\partial t} \mathbf{u}(\tau)\right\|^{2}-\frac{1}{2}\left\|\frac{\partial}{\partial t} \mathbf{u}(0)\right\|^{2}+\int_{0}^{\tau}\left\langle\mathbf{T}(t), \nabla \frac{\partial}{\partial t} \mathbf{u}(\mathbf{t})\right\rangle d t=0 .
$$

Since $\mathbf{T}$ is symmetric and $\mathbf{E}=\operatorname{sym} \nabla \mathbf{u}$, by virtue of $(2.2)$ and Theorem 1 the following inequality holds:

$$
\int_{0}^{\tau}\left\langle\mathbf{T}(t), \nabla \frac{\partial}{\partial t} \mathbf{u}(t)\right\rangle d t=\int_{0}^{\tau}\left\langle\mathbf{T}(t), \frac{\partial}{\partial t} \mathbf{E}(t)\right\rangle d t \geq\left\|\nabla \mathbf{u}^{\tau}\right\|\left\|_{\mathbf{N}}^{2}-\right\| \nabla \mathbf{u}^{0}\|\|_{\mathbf{N}}^{2}
$$

where

$$
\left\|\nabla \mathbf{u}^{t}\right\|_{\mathbf{N}}^{2}=\int_{\Omega} \Psi_{\mathbf{N}}\left(\mathbf{E}(t),{ }_{r} \mathbf{E}^{t}\right) d \mathbf{x}=\int_{\Omega}\left\|\nabla \mathbf{u}^{t}\right\|_{\mathbf{N}}^{2} d \mathbf{x} .
$$

Therefore, from (2.7) it follows that

$$
\frac{1}{2}\left\|\frac{\partial}{\partial t} \mathbf{u}(\tau)\right\|^{2}+\left.\left\|\nabla \nabla \mathbf{u}^{\tau}\right\|\right|_{\mathbf{N}} ^{2} \leq \frac{1}{2}\left\|\frac{\partial}{\partial t} \mathbf{u}(0)\right\|^{2}+\left\|\nabla \mathbf{u}^{0}\right\| \|_{\mathbf{N}}^{2}
$$

Now, the total mechanical energy associated to each specific free energy $\Psi_{\mathbf{N}}$, i.e.,

$$
\mathcal{E}_{\mathbf{N}}\left(\mathbf{u}^{t}\right)=\int_{\Omega}\left[\frac{1}{2}\left|\frac{\partial}{\partial t} \mathbf{u}^{t}(0)\right|^{2}+\Psi_{\mathbf{N}}\left(\mathbf{E}(t),{ }_{r} \mathbf{E}^{t}\right)\right] d \mathbf{x}
$$

can be viewed as the $\mathbf{N}$-energy norm, namely,

$$
\left\|\mid \mathbf{u}^{t}\right\|\left\|_{\mathcal{E}_{\mathbf{N}}}^{2}=\mathcal{E}_{\mathbf{N}}\left(\mathbf{u}^{t}\right)=\frac{1}{2}\right\| \frac{\partial}{\partial t} \mathbf{u}^{t}(0)\left\|^{2}+\right\| \nabla \mathbf{u}^{t} \|_{\mathbf{N}}^{2} .
$$

\footnotetext{
${ }^{3}$ Let $\langle\cdot, \cdot\rangle$ be the usual spatial $L^{2}$ inner product, namely $\langle\mathbf{u}, \mathbf{v}\rangle=\operatorname{Re} \int_{\Omega} \mathbf{u}(\mathbf{x}) \cdot \mathbf{v}^{*}(\mathbf{x}) d \mathbf{x}$, where $\mathbf{v}^{*}$ is the conjugate of $\mathbf{v}$. The corresponding $L^{2}$-norm is denoted by $\|\cdot\|$, so that $\|\mathbf{u}\|^{2}=\langle\mathbf{u}, \mathbf{u}\rangle$.
} 
Then, by (2.9), each total-energy functional is nonincreasing along any solution, and the continuity property of $\mathcal{E}_{\mathrm{N}}$ yields the following

TheOREM 2. Under the assumptions of Theorem 1, the mechanical energy is a Lyapunov functional for the evolutive system (2.6). Indeed, $\mathcal{E}_{\mathrm{N}}$ is

- continuous with respect to the norm \|\|$\cdot \|\left.\right|_{\mathcal{E}_{\mathbf{N}}}$,

- nonnegative for any $\mathbf{u}^{t}$ and vanishing if and only if $\mathbf{u}^{t}=\mathbf{0}^{\dagger}$,

- not increasing along any solution of (2.6), i.e., $\mathcal{E}_{\mathbf{N}}\left(\mathbf{u}^{t}\right) \leq \mathcal{E}_{\mathbf{N}}\left(\mathbf{u}^{0}\right), t \geq 0$.

Relative to each $\mathbf{N}$-energy norm, this theorem provides the zero solution to be stable with respect to the corresponding class of perturbations

$$
\mathcal{U}_{\mathbf{N}}^{0}=\left\{\mathbf{u}^{0}: \mathbb{R}^{+} \rightarrow H_{0}^{1}(\Omega) \text { such that }\|\| \mathbf{u}^{0} \|_{\mathcal{E}_{\mathbf{N}}}<\infty\right\}
$$

Remarkably, $\mathcal{E}_{1}$ is defined for any relaxation function $\mathbf{G}$ compatible with $\mathbb{P}_{1}-\mathbb{P}_{5}$ and, by virtue of $(2.5)$ and Remark 1 , for each $\mathcal{E}_{\mathbf{N}}$ it follows that

$$
\mathcal{U}_{\mathbf{1}}^{0} \subset \mathcal{U}_{\mathbf{N}}^{0} \quad \text { and } \quad\left\|\left|\mathbf{u}^{0}\left\|\left.\right|_{\mathcal{E}_{\mathbf{N}}} \leq\right\|\left\|\mathbf{u}^{0}\right\|_{\mathcal{E}_{1}} \quad \text { for all } \mathbf{u}^{0} \in \mathcal{U}_{\mathbf{1}}^{0}\right.\right.
$$

Namely, $\mathcal{U}_{1}^{0}$ identifies the widest set of perturbations such that the solution is stable relative to every energy norm. As a consequence of Remark 2, unfortunately, this set contains no oscillating past-histories. If $\mathbf{G}^{\prime}<\mathbf{0}$ and $\mathbf{G}^{\prime \prime} \geq \mathbf{0}$, then the choice $\mathbf{N}=$ $\left(-\mathbf{G}_{s}^{\prime}\right)^{1 / 2}$ leads to Graffi-Volterra's total energy, $\mathcal{E}_{\mathbf{G}}$, and the corresponding norm ensures stability with respect to all bounded perturbations.

Finally, it is worth noting that stability relative to any $\mathbf{N}$-energy norm implies stability relative to the usual elastic-energy norm. By use of (2.3), (2.5), and (2.10), property $\mathbb{P}_{3}$ along with the Korn-Poincaré inequality provide

$$
\left\|\mathbf{u}^{t}\right\| \|_{\mathcal{E}_{\mathbf{N}}}^{2} \geq \frac{1}{2}\left[\left\|\frac{\partial}{\partial t} \mathbf{u}(t)\right\|^{2}+\left\langle\nabla \mathbf{u}(t), \mathbf{G}_{\infty} \nabla \mathbf{u}(t)\right\rangle\right] \geq \frac{1}{2}\left[\left\|\frac{\partial}{\partial t} \mathbf{u}(t)\right\|^{2}+\beta\|\mathbf{u}(t)\|_{H^{1}(\Omega)}^{2}\right]
$$

Therefore, for any $\mathbf{N}$ (compatible with $\mathbb{A}_{1}-\mathbb{A}_{2}$ ) Theorem 2 leads to "classical" stability of the null solution, but with respect to different sets of initial perturbations, namely

$$
\left\|\frac{\partial}{\partial t} \mathbf{u}(t)\right\|+\|\mathbf{u}(t)\|_{H^{1}(\Omega)} \leq c\|\| \mathbf{u}^{t}\|\|_{\mathcal{E}_{\mathbf{N}}} \leq c\|\| \mathbf{u}^{0}\|\|_{\mathcal{E}_{\mathbf{N}}}
$$

3. Stability via "a priori" inequalities. In this section the same assumptions $\mathbb{P}_{1}-\mathbb{P}_{5}$ are involved; however, following a different method previous stability results are improved. With reference to an energy-like norm, all solutions of (1.8) prove to be stable with respect to a class $\mathcal{U}$ of initial past-history perturbations larger than $\mathcal{U}_{1}^{0}$.

Remarkably, set $\mathcal{U}$ is affected by the asymptotic behaviour of the constitutive function $\mathbf{G}^{\prime}$, whereas $\mathcal{U}_{1}^{0}$ is not. In particular, $\mathcal{U}$ might contain all bounded initial past-histories if $\mathbf{G}^{\prime}$ were properly decaying to zero as $t \rightarrow \infty$. 
In order to emphasize the dependence on the initial perturbation $\mathbf{u}^{0}$, the given problem (2.6) may be rewritten as follows:

$$
\begin{cases}\frac{\partial^{2}}{\partial t^{2}} \mathbf{u}(t)-\nabla \cdot\left[\mathbf{G}_{0} \nabla \mathbf{u}(t)+\int_{0}^{t} \mathbf{G}^{\prime}(s) \nabla \mathbf{u}(t-s) d s\right]=\nabla \cdot \mathbf{T}_{0}(t) \quad \text { on } \Omega \times(0, \tau), \\ \mathbf{u}(t)=\mathbf{0}, & \text { on } \partial \Omega \times(0, \tau), \\ \mathbf{u}(0)=\mathbf{u}_{0}, \quad \frac{\partial}{\partial t} \mathbf{u}(0)=\mathbf{v}_{0}, & \text { on } \Omega,\end{cases}
$$

where the source term and the initial data depend on $\mathbf{u}^{0}$, namely,

$$
\mathbf{T}_{0}(t)=\int_{0}^{\infty} \mathbf{G}^{\prime}(t+s) \nabla \mathbf{u}^{0}(s) d s, \quad \mathbf{u}_{0}=\mathbf{u}^{0}\left(0^{+}\right), \quad \mathbf{v}_{0}=-\frac{\partial}{\partial s} \mathbf{u}^{0}\left(0^{+}\right)
$$

Let us introduce the set of perturbations $\mathcal{U}$ as follows:

Definition 3. $\mathcal{U}$ is the set of initial past-history $\mathbf{u}^{0}: \mathbb{R}^{+} \rightarrow H_{0}^{1}(\Omega)$ such that

i) $\frac{\partial}{\partial s} \mathbf{u}^{0}\left(0^{+}\right) \in L^{2}(\Omega)$;

ii) $\frac{\partial}{\partial t} \mathbf{T}_{0} \in L^{1}\left(\mathbb{R}^{+}, L^{2}(\Omega)\right)$ and $\mathbf{T}_{0}(0) \in L^{2}(\Omega)$, or

ii) $)_{2} \nabla \cdot \mathbf{T}_{0} \in L^{1}\left(\mathbb{R}^{+}, L^{2}(\Omega)\right)$ and $\mathbf{u}^{0}\left(0^{+}\right) \in H^{2}(\Omega)$.

Assuming $\mathbb{P}_{1}-\mathbb{P}_{5}$ hold, we prove the following a priori inequality:

Theorem 3. When $\mathbf{u}^{0} \in \mathcal{U}$ the corresponding solution $\mathbf{u}: \mathbb{R}^{+} \rightarrow H_{0}^{1}(\Omega)$ to (3.1) satisfies

$$
\left\|\frac{\partial}{\partial t} \mathbf{u}(\tau)\right\|^{2}+\|\mathbf{u}(\tau)\|_{H^{1}(\Omega)}^{2}+\|\nabla \mathbf{u}\|_{\mathbf{1}}^{2} \leq c_{0} \quad \text { for any } \tau>0
$$

where $c_{0}$ only depends on suitable norms of $\mathbf{u}_{0}, \mathbf{v}_{0}, \mathbf{T}_{0}(0)$, and $\frac{\partial}{\partial t} \mathbf{T}_{0}$ or $\nabla \cdot \mathbf{T}_{0}$.

Proof. $\mathbf{u}(t), t>0$, being the unknown function of (3.1), we set

$$
\mathbf{v}=\mathbf{u}-\varphi \mathbf{u}_{0}
$$

where $\varphi$ is a rapidly decreasing function such that $0 \leq \varphi(t) \leq \varphi(0)=1$ and $\varphi^{\prime}(0)=0$. Both $\mathbf{u}$ and $\mathbf{v}$ have the same asymptotic behaviour as $t \rightarrow+\infty$, but $\mathbf{v}(0)=\mathbf{0}$ and $\frac{\partial}{\partial t} \mathbf{v}(0)=\mathbf{v}_{0}$.

In order to extend $\mathbf{v}$ preserving its continuity at 0 , we identify $\mathbf{v}$ on $[0, \tau]$ with the corresponding function on $(-\infty, \tau]$ which vanishes identically on $(-\infty, 0]$. Thereby, a straightforward calculation yields

$$
\mathbf{G}_{0} \nabla \mathbf{v}(t)+\int_{0}^{t} \mathbf{G}^{\prime}(s) \nabla \mathbf{v}(t-s) d s=\mathbf{G}_{\infty} \nabla \mathbf{v}(t)+\int_{0}^{\infty} \mathbf{G}^{\prime}(s)[\nabla \mathbf{v}(t-s)-\nabla \mathbf{v}(t)] d s
$$

and, in terms of $\mathbf{v},(3.1)$ takes the form

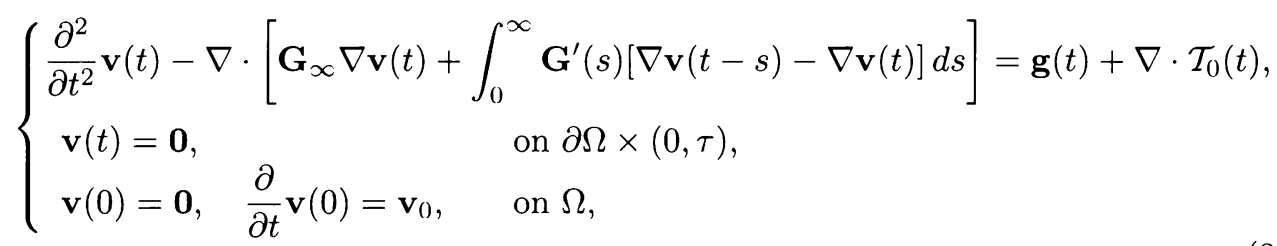

where

$$
\mathbf{g}(t)=-\varphi^{\prime \prime}(t) \mathbf{u}_{0}, \quad \mathcal{T}_{0}(t)=\mathbf{T}_{0}(t)+\left[\mathbf{G}_{0} \varphi(t)+\int_{0}^{t} \mathbf{G}^{\prime}(s) \varphi(t-s) d s\right] \nabla \mathbf{u}_{0} .
$$


The corresponding theorem of expended power reads

$$
\begin{aligned}
\frac{1}{2}\left\|\frac{\partial}{\partial t} \mathbf{v}(\tau)\right\|^{2}+ & \frac{1}{2}\left\langle\nabla \mathbf{v}(\tau), \mathbf{G}_{\infty} \nabla \mathbf{v}(\tau)\right\rangle \\
& +\int_{0}^{\tau}\left\langle\int_{0}^{\infty} \mathbf{G}^{\prime}(s)[\nabla \mathbf{v}(t-s)-\nabla \mathbf{v}(t)] d s, \nabla \frac{\partial}{\partial t} \mathbf{v}(t)\right\rangle d t \\
=\frac{1}{2}\left\|\mathbf{v}_{0}\right\|^{2} & +\int_{0}^{\tau}\left\langle\mathbf{g}(t), \frac{\partial}{\partial t} \mathbf{v}(t)\right\rangle d t+\int_{0}^{\tau}\left\langle\nabla \cdot \mathcal{T}_{0}(t), \frac{\partial}{\partial t} \mathbf{v}(t)\right\rangle d t
\end{aligned}
$$

or else, after integration by parts in $\mathbf{x}$ and $t$ of the last integral,

$$
\begin{aligned}
\frac{1}{2}\left\|\frac{\partial}{\partial t} \mathbf{v}(\tau)\right\|^{2}+ & \frac{1}{2}\left\langle\nabla \mathbf{v}(\tau), \mathbf{G}_{\infty} \nabla \mathbf{v}(\tau)\right\rangle \\
& +\int_{0}^{\tau}\left\langle\int_{0}^{\infty} \mathbf{G}^{\prime}(s)[\nabla \mathbf{v}(t-s)-\nabla \mathbf{v}(t)] d s, \nabla \frac{\partial}{\partial t} \mathbf{v}(t)\right\rangle d t \\
= & \frac{1}{2}\left\|\mathbf{v}_{0}\right\|^{2}+\int_{0}^{\tau}\left\langle\mathbf{g}(t), \frac{\partial}{\partial t} \mathbf{v}(t)\right\rangle d t+\int_{0}^{\tau}\left\langle\frac{\partial}{\partial t} \mathcal{T}_{0}(t), \nabla \mathbf{v}(t)\right\rangle d t-\left\langle\mathcal{T}_{0}(\tau), \nabla \mathbf{v}(\tau)\right\rangle
\end{aligned}
$$

On the basis of Parseval's Lemma, some manipulations allow us to transform the double integral on the left-hand side of $(3.4)_{1}$ and $(3.4)_{2}$ into a quadratic form. Actually, we obtain

$$
\begin{aligned}
\int_{0}^{\tau}\left\langle\int_{0}^{\infty} \mathbf{G}^{\prime}(s)[\nabla \mathbf{v}(t\right. & \left.-s)-\nabla \mathbf{v}(t)] d s, \frac{\partial}{\partial t} \nabla \mathbf{v}(t)\right\rangle d t \\
= & -\frac{1}{\pi} \int_{0}^{\infty}\left\langle\omega \mathbf{G}_{s}^{\prime}(\omega) \nabla \hat{\mathbf{v}}_{\tau}(\omega), \nabla \hat{\mathbf{v}}_{\tau}(\omega)\right\rangle d \omega
\end{aligned}
$$

where $\mathbf{v}_{\tau}$ is defined by

$$
\mathbf{v}_{\tau}(t)= \begin{cases}\mathbf{v}(t), & t \in(-\infty, \tau] \\ \mathbf{v}(\tau)=\mathbf{u}(\tau)-\varphi(\tau) \mathbf{u}_{0}, & t \in(\tau,+\infty)\end{cases}
$$

namely, $\mathbf{v}_{\tau}$ vanishes on $(-\infty, 0)$, is constant on $(\tau,+\infty)$ and is continuous across 0 and $\tau$. Then, the restriction of $\mathbf{v}_{\tau}$ on $\mathbb{R}^{+}$may be handled as a continuous history with vanishing initial value and, in connection with the notation of Sec. 2 , we may set

$$
\left\|\mid \nabla \mathbf{v}_{\tau}\right\|_{1}^{2}=-\frac{1}{\pi} \int_{0}^{\infty}\left\langle\omega \mathbf{G}_{s}^{\prime}(\omega) \nabla \hat{\mathbf{v}}_{\tau}(\omega), \nabla \hat{\mathbf{v}}_{\tau}(\omega)\right\rangle d \omega
$$


Accordingly, because of property $\mathbb{P}_{3}$ and Korn's inequality, from $(3.4)_{1}$ and $(3.4)_{2}$ respectively follow

$$
\begin{aligned}
& \frac{1}{2}\left[\left\|\frac{\partial}{\partial t} \mathbf{v}(\tau)\right\|^{2}+\beta\|\nabla \mathbf{v}(\tau)\|^{2}\right]+\left\|\nabla \mathbf{v}_{\tau}\right\|_{1}^{2} \\
& \quad \leq \frac{1}{2}\left\|\mathbf{v}_{0}\right\|^{2}+\int_{0}^{\tau}\left[\|\mathbf{g}(t)\|+\left\|\nabla \cdot \mathcal{T}_{0}(t)\right\|\right]\left\|\frac{\partial}{\partial t} \mathbf{v}(t)\right\| d t \\
& \frac{1}{2}\left[\left\|\frac{\partial}{\partial t} \mathbf{v}(\tau)\right\|^{2}+\beta\|\nabla \mathbf{v}(\tau)\|^{2}\right]+\left\|\nabla \mathbf{v}_{\tau}\right\|\left\|_{\mathbf{1}}^{2} \leq \frac{1}{2}\right\| \mathbf{v}_{0}\left\|^{2}+\int_{0}^{\tau}\right\| \mathbf{g}(t)\|\| \frac{\partial}{\partial t} \mathbf{v}(t) \| d t \\
& \quad+\int_{0}^{\tau}\left\|\frac{\partial}{\partial t} \mathcal{T}_{0}(t)\right\|\|\nabla \mathbf{v}(t)\| d t+\frac{1}{2 \varepsilon}\left\|\mathcal{T}_{0}(\tau)\right\|^{2}+\frac{\varepsilon}{2}\|\nabla \mathbf{v}(\tau)\|^{2} .
\end{aligned}
$$

Previous inequalities are exploited by means of a Gronwall-type lemma (see, e.g., [12]):

Lemma 1 . Let $g$ be continuous and positive on $[0, t], t>0$. If a constant $C \geq 0$ and two nonnegative functions $\alpha, \beta \in L^{1}(0, t)$ exist such that

$$
0 \leq g(s) \leq C+2 \int_{0}^{s} \alpha(r) \sqrt{g(r)} d r+\int_{0}^{s} \beta(r) g(r) d r \quad \text { for all } s \in(0, t)
$$

then it necessarily follows that

$$
g(t) \leq\left[\sqrt{C}+\int_{0}^{t} \alpha(s) d s\right]^{2} \exp \left[\int_{0}^{t} \beta(s) d s\right]
$$

Inequality $(3.7)_{1}$ immediately yields

$$
\frac{1}{2}\left[\left\|\frac{\partial}{\partial t} \mathbf{v}(\tau)\right\|^{2}+\beta\|\nabla \mathbf{v}(\tau)\|^{2}\right]+\|\| \nabla \mathbf{v}_{\tau} \|_{1}^{2} \leq\left\{\left\|\mathbf{v}_{0}\right\|+\int_{0}^{\infty}\left[\|\mathbf{g}(t)\|+\left\|\nabla \cdot \mathcal{T}_{0}(t)\right\|\right] d t\right\}^{2} .
$$

On the other hand, choosing $\varepsilon$ small enough, inequality $(3.7)_{2}$ yields

$$
\begin{aligned}
\frac{1}{2}\left[\left\|\frac{\partial}{\partial t} \mathbf{v}(\tau)\right\|^{2}+\gamma^{2}\|\nabla \mathbf{v}(\tau)\|^{2}\right] & +\left\|\nabla \mathbf{v}_{\tau}\right\|_{1}^{2} \leq \frac{1}{2 \varepsilon}\left[\left\|\mathbf{v}_{0}\right\|^{2}+\left\|\mathcal{T}_{0}(\tau)\right\|^{2}\right] \\
& +\int_{0}^{\tau}\|\mathbf{g}(t)\|\left\|\frac{\partial}{\partial t} \mathbf{v}(t)\right\| d t+\int_{0}^{\tau}\left\|\frac{\partial}{\partial t} \mathcal{T}_{0}(t)\right\|\|\nabla \mathbf{v}(t)\| d t
\end{aligned}
$$

and, by means of trivial manipulations, it follows that

$$
\begin{aligned}
& {\left[\left\|\frac{\partial}{\partial t} \mathbf{v}(\tau)\right\|+\gamma\|\nabla \mathbf{v}(\tau)\|\right]^{2}+4\|\| \nabla \mathbf{v}_{\tau}\|\|_{\mathbf{1}}^{2} \leq \frac{2}{\varepsilon}\left[\left\|\mathbf{v}_{0}\right\|^{2}+\left\|\mathcal{T}_{0}(\tau)\right\|^{2}\right]} \\
& +4 \int_{0}^{\tau}\left\{\|\mathbf{g}(t)\|+\frac{1}{\gamma}\left\|\frac{\partial}{\partial t} \mathcal{T}_{0}(t)\right\|\right\}\left\{\left\|\frac{\partial}{\partial t} \mathbf{v}(t)\right\|+\gamma\|\nabla \mathbf{v}(t)\|\right\} d t
\end{aligned}
$$


Finally, applying the previous Gronwall-type lemma, we obtain

$$
\begin{aligned}
& \frac{1}{2}\left[\left\|\frac{\partial}{\partial t} \mathbf{v}(\tau)\right\|^{2}+\gamma^{2}\|\nabla \mathbf{v}(\tau)\|^{2}\right]+\left\|\nabla \mathbf{v}_{\tau}\right\| \|_{1}^{2} \\
& \quad \leq c_{1}\left[\left\|\mathbf{v}_{0}\right\|^{2}+\|\| \mathcal{T}_{0} \|_{\infty, 2}^{2}\right]+c_{2}\left\{\int_{0}^{\infty}\left[\|\mathbf{g}(t)\|+\left\|\frac{\partial}{\partial t} \mathcal{T}_{0}(t)\right\|\right] d t\right\}^{2}
\end{aligned}
$$

where $c_{1}, c_{2}$ are positive constants depending on $\gamma, \varepsilon$ and $\|\mid \cdot\| \|_{k, 2}$ denotes the norm of $L^{k}\left(\mathbb{R}^{+}, L^{2}(\Omega)\right)$, for $k=1,2, \ldots, \infty$. Now, taking into account (3.6) and the expressions of $\mathbf{v}$ and $\mathbf{v}_{\tau}$ in terms of $\mathbf{u}$, a straightforward calculation yields

$$
\begin{aligned}
&\|\nabla \mathbf{u}(\tau)\| \leq\|\nabla \mathbf{v}(\tau)\|+\left\|\nabla \mathbf{u}_{0}\right\|, \quad\|\nabla \mathbf{u}\|_{1} \leq\left.\sup _{t \in \mathbb{R}^{+}}\left\|\nabla \mathbf{v}_{\tau}\right\|\right|_{1} ^{2}+k_{1}\left\|\mathbf{u}_{0}\right\|_{H^{1}(\Omega)}, \\
&\left\|\frac{\partial}{\partial t} \mathbf{u}(t)\right\| \leq\left\|\frac{\partial}{\partial t} \mathbf{v}(\tau)\right\|+k_{2}\left\|\mathbf{u}_{0}\right\|, \quad\left\|\frac{\partial}{\partial t} \mathcal{T}_{0}\right\|\left\|_{1,2} \leq\right\|\left\|\frac{\partial}{\partial t} \mathcal{T}_{0}\right\|\left\|_{1,2}+k_{3}\right\| \mathbf{u}_{0} \|_{H^{1}(\Omega)} \\
&\|\mathbf{g}\|_{1,2} \leq k_{2}\left\|\mathbf{u}_{0}\right\|,\left\|\nabla \cdot \mathcal{T}_{0}\right\|\left\|_{1,2} \leq\right\| \nabla \cdot \mathcal{T}_{0}\left\|_{1,2}+k_{4}\right\| \mathbf{u}_{0} \|_{H^{2}(\Omega)} \\
&\left\|\mathcal{T}_{0}\right\|_{\infty, 2} \leq\left\|\frac{\partial}{\partial t} \mathcal{T}_{0}\right\|\left\|_{1,2}+\right\| \mathcal{T}_{0}(0)\|\leq\| \frac{\partial}{\partial t} \mathbf{T}_{0}\|\|_{1,2}+k_{5}\left\|\mathbf{u}_{0}\right\|_{H^{1}(\Omega)}+\left\|\mathbf{T}_{0}(0)\right\|
\end{aligned}
$$

where $k_{1}, k_{2}, k_{3}, k_{4}, k_{5}$ are positive constants depending on $\varphi, \mathbf{G}$, and $\Omega$. Then, by Definition 3 and the Poincaré inequality, as a consequence of inequalities $(3.8)_{1}-(3.8)_{2}$ the thesis easily follows.

Finally, we point out that $\mathcal{U}$ is affected by the asymptotic behaviour of the relaxation function. Taking the homogeneous case into account, for the sake of simplicity, $\mathcal{U}$ contains all bounded past-histories if $\mathbf{G}^{\prime}$ is properly decaying to zero as $t \rightarrow \infty$.

This feature may be emphasized by means of the following

REMARK 4. Any initial past-histories $\mathbf{u}^{0} \in L^{\infty}\left(\mathbb{R}^{+}, H_{0}^{1}(\Omega) \cap H^{2}(\Omega)\right)$ satisfy ii $)_{2}$ of Definition 3 provided that $\mathbf{G}^{\prime}$ obeys the condition

$$
\int_{0}^{\infty} \int_{0}^{\infty}\left|\mathbf{G}^{\prime}(s+t)\right| d s d t<+\infty
$$

On the other hand, any $\mathbf{u}^{0} \in L^{\infty}\left(\mathbb{R}^{+}, H_{0}^{1}(\Omega)\right)$ satisfies ii) $)_{1}$ of Definition 3 if

$$
\int_{0}^{\infty} \int_{0}^{\infty}\left|\mathbf{G}^{\prime \prime}(s+t)\right| d s d t<+\infty
$$


Proof. By use of the definition of $\mathbf{T}_{0}$, we can easily obtain the following inequalities:

$$
\begin{aligned}
\left\|\nabla \cdot \mathbf{T}_{0}(t)\right\| \|_{1,2} & \leq \int_{0}^{\infty} \int_{0}^{\infty}\left\|\nabla \cdot\left[\mathbf{G}^{\prime}(t+s) \nabla \mathbf{u}^{0}(s)\right]\right\| d s d t \\
& \leq \kappa_{1} \int_{0}^{\infty} \int_{0}^{\infty}\left|\mathbf{G}^{\prime}(t+s)\right|\left\|\mathbf{u}^{0}(s)\right\|_{H^{2}(\Omega)} d s d t \\
\|\| \frac{\partial}{\partial t} \mathbf{T}_{0}(t)\|\|_{1,2} & \leq \int_{0}^{\infty} \int_{0}^{\infty}\left\|\mathbf{G}^{\prime \prime}(t+s) \nabla \mathbf{u}^{0}(s)\right\| d s d t \\
& \leq \kappa_{2} \int_{0}^{\infty} \int_{0}^{\infty}\left|\mathbf{G}^{\prime \prime}(t+s)\right|\left\|\mathbf{u}^{0}(s)\right\|_{H^{1}(\Omega)} d s d t \\
\left\|\mathbf{T}_{0}(0)\right\| & \leq \int_{0}^{\infty}\left\|\mathbf{G}^{\prime}(s) \nabla \mathbf{u}^{0}(s)\right\| d s \leq \kappa_{2} \int_{0}^{\infty}\left|\mathbf{G}^{\prime}(s)\right|\left\|\mathbf{u}^{0}(s)\right\|_{H^{1}(\Omega)} d s
\end{aligned}
$$

that provide both conditions (a) and (b).

It is worth noting that (a) and (b) compel the asymptotic decay of $\mathbf{G}^{\prime}$ in a customary fashion. For instance, (a) is satisfied by any $\mathbf{G}^{\prime} \in L^{1}\left(\mathbb{R}^{+}\right)$that meets one of the following properties:

i) $\left|\mathbf{G}^{\prime}(t+s)\right| \leq k\left|\mathbf{G}^{\prime}(t)\right|\left|\mathbf{G}^{\prime}(s)\right|$,

ii) $\left|\mathbf{G}^{\prime}(t)\right| \leq k(1+t)^{-2-\varepsilon}$ with $k, \varepsilon>0$,

iii) $\mathbf{G}^{\prime}(t) \leq \mathbf{0}$ and $\mathbf{G}-\mathbf{G}_{\infty} \in L^{1}\left(\mathbb{R}^{+}\right)$.

Similarly, (b) is verified by any absolutely continuous function $\mathbf{G}^{\prime} \in L^{1}\left(\mathbb{R}^{+}\right)$obeying

$$
\left|\mathbf{G}^{\prime \prime}(t)\right| \leq \frac{k}{(1+t)^{2+\varepsilon}} \quad(k, \varepsilon>0)
$$

or

$$
\mathbf{G}^{\prime}(t) \leq \mathbf{0} \quad \text { and } \quad \mathbf{G}^{\prime \prime}(t) \geq \mathbf{0}
$$

4. Asymptotic stability. Besides achieving stability, assumptions $\mathbb{P}_{1}-\mathbb{P}_{4}$ were also successfully applied to prove well-posedness of both dynamical (on the whole time axis) and quasi-static boundary value problems (see $[13,14]$ ). Nevertheless, we notice that assumption $\mathbb{P}_{4}$ might appear too restrictive. From a mathematical point of view, indeed, it is sufficient but not necessary to ensure the existence of a unique solution to the quasi-static problem [15]. Moreover, $\mathbb{P}_{4}$ along with $\mathbb{P}_{3}$ turns out to be stronger than the "strong dissipation principle" stated by Gurtin and Herrera [16].

In spite of this, for a linear viscoelastic solid obeying $\mathbb{P}_{1}-\mathbb{P}_{3}$, condition $\mathbb{P}_{4}$ provides a necessary and sufficient condition to gain asymptotic stability of solutions to (1.8). First, in order to prove this statement, we recall the following result.

Theorem 4 ([11], Theorem 1). Let us assume that the relaxation function $\mathbf{G}$ satisfies $\mathbb{P}_{1}-\mathbb{P}_{4}$. If the initial past-history $\mathbf{u}^{0}$ satisfies i) of Definition 3 and, moreover, is such that $\nabla \cdot T_{0}(\mathbf{x}, t)$ belongs to $L^{2}\left(\mathbb{R}^{+}, H^{-1}(\Omega)\right)$, then problem $(3.1)$ has one and only one solution

$$
\mathbf{u} \in H^{1}\left(\mathbb{R}^{+}, L^{2}(\Omega)\right) \cap L^{2}\left(\mathbb{R}^{+}, H_{0}^{1}(\Omega)\right)
$$


By joining this Theorem to Theorem 3 , we can easily conclude that $\mathbb{P}_{1}-\mathbb{P}_{5}$ provide sufficient conditions to achieve asymptotic stability (in the ordinary sense). This is stated in the following result.

Theorem 5. Let us assume that the relaxation function $\mathbf{G}$ satisfies $\mathbb{P}_{1}-\mathbb{P}_{5}$ and the initial past-history $\mathbf{u}^{0}$ belongs to $\mathcal{U}^{*}$, where

$$
\mathcal{U}^{*}=\left\{\mathbf{u}^{0} \in \mathcal{U}: \nabla \cdot T_{0} \in L^{2}\left(\mathbb{R}^{+}, H^{-1}(\Omega)\right)\right\} .
$$

Then (3.1) has one and only one solution $\mathbf{u}$ such that

$$
\mathbf{u} \in L^{2}\left(\mathbb{R}^{+}, H_{0}^{1}(\Omega)\right) \cap L^{\infty}\left(\mathbb{R}^{+}, H_{0}^{1}(\Omega)\right)
$$

and

$$
\frac{\partial}{\partial t} \mathbf{u} \in L^{2}\left(\mathbb{R}^{+}, L^{2}(\Omega)\right) \cap L^{\infty}\left(\mathbb{R}^{+}, L^{2}(\Omega)\right)
$$

In order to prove the necessity of condition $\mathbb{P}_{4}$ we have to show that the above Theorem fails if inequality (1.3) is weakened. In particular, we assume that a circular frequency $\omega^{*}>0$ and a corresponding tensor $\mathbf{A}^{*} \in \operatorname{Sym} \backslash\{\mathbf{0}\}$ exist such that

$\mathbb{P}_{4}^{\prime} \cdot \mathbf{G}_{s}^{\prime}(\omega)<0 \quad$ for any $\omega \in \mathbb{R}^{++} \backslash\left\{\omega^{*}\right\}$ and $\mathbf{A}^{*} \cdot \mathbf{G}^{\prime}{ }_{s}\left(\omega^{*}\right) \mathbf{A}^{*}=0$.

Remarkably, condition $\mathbb{P}_{4}^{\prime}$ is allowed by the negative semidefiniteness of $\mathbf{G}_{s}^{\prime}$ that is quite equivalent to the Second Law of Thermodynamics in the form of the Clausius property for isothermal cyclic processes (see [4]). In this connection, as suggested by Fabrizio [14], the stronger form $\mathbb{P}_{4}$ of such a thermodynamic inequality is justified by observing that the statement of the Second Law in standard textbooks requires the distinction between reversible and irreversible processes and specifies that the equality sign refers to the former ones and the inequality sign to the latter ones. Roughly speaking, in a linear viscoelastic solid the process associated with any nontrivial (i.e., not constant) strain-history is assumed to be "irreversible", thus involving the strict inequality.

If $\mathbb{P}_{4}$ is weakened into $\mathbb{P}_{4}^{\prime}$ then the asymptotic stability property no longer holds. For the sake of simplicity, henceforth, let us restrict our attention to one-dimensional, homogeneous viscoelastic solids. Accordingly, constitutive equation (1.1) takes the form

$$
\boldsymbol{T}\left(u_{x}^{t}(\mathbf{x}, \cdot)\right)=\mathbf{G}_{0} u_{x}^{t}(\mathbf{x}, 0)+\int_{0}^{\infty} \mathbf{G}^{\prime}(s) u_{x}^{t}(\mathbf{x}, s) d s, \quad x \in \Omega=(0, l)
$$

and (1.8) leads to the following boundary initial-history value problem:

$$
\left\{\begin{aligned}
u_{t t}(\mathbf{x}, t)-\mathbf{G}_{0} u_{x x}(\mathbf{x}, t)-\int_{0}^{\infty} \mathbf{G}^{\prime}(s) u_{x x}(\mathbf{x}, t-s) d s=f(\mathbf{x}, t), & (\mathbf{x}, t) \in(0, l) \times \mathbb{R}^{+}, \\
u(\mathbf{x}, t)=u^{0}(\mathbf{x},-t), & (\mathbf{x}, t) \in(0, l) \times \mathbb{R}^{-}, \\
u(0, t)=u(l, t)=0, & t \in \mathbb{R}^{+} .
\end{aligned}\right.
$$

Here $\mathbf{G}^{\prime}$ is assumed to be any (scalar) function obeying $\mathbb{P}_{1}, \mathbb{P}_{2}, \mathbb{P}_{4}^{\prime}, \mathbb{P}_{5}$ and, moreover, $\mathbf{G}_{\infty}=\mathbf{G}_{0}+\int_{0}^{\infty} \mathbf{G}^{\prime}(s) d s$ is chosen to be positive, in agreement with $\mathbb{P}_{3}$.

The following Theorem shows that such relaxation functions compel all solutions of (4.2) to be stable, but not asymptotically stable. Namely, if $l$ is properly chosen, there exists a family of sinusoidal-in-time initial past-history which supports oscillating solutions of the same frequency $\omega^{*}$. Hence, the null solution of the corresponding homogeneous 
problem with nonzero initial data fails to be attractive, and the lack of asymptotic stability does occur. From a physical point of view, this allows the body to behave elastically at some prescribed frequency. Such a situation is quite strange for a viscoelastic material, where dissipation takes place along every nontrivial process.

Theorem 6. Let $G^{\prime}$ be any Boltzmann function agreeing with $\mathbb{P}_{1}, \mathbb{P}_{2}, \mathbb{P}_{4}^{\prime}, \mathbb{P}_{5}$ and obeying property (a) of Remark 4 . Let $\mathbf{G}_{x}^{*}, l^{*}$, and $u^{*}$ be such that

$$
\begin{gathered}
\mathbf{G}_{\infty}^{*}>0 \text { and } \mathbf{G}_{\infty}^{*}>\int_{0}^{\infty} \mathbf{G}^{\prime}(s)\left(1-\cos \omega^{*} s\right) d s, \\
l^{*}=\frac{\sqrt{\mathbf{G}_{0}^{*}+\mathbf{G}_{c}^{\prime}\left(\omega^{*}\right)}}{\omega^{*}} \pi, \\
u^{*}(\mathbf{x}, t)=c \sin \left(\frac{\pi \mathbf{x}}{l^{*}}\right)\left(\cos \omega^{*} t+\sin \omega^{*} t\right), \quad t \in \mathbb{R}^{+} .
\end{gathered}
$$

Then, for any $c \in \mathbb{R}$, the history $u^{*}$ belongs to $\mathcal{U}^{*}$ and the homogeneous problem

$$
\begin{cases}u_{t t}(\mathbf{x}, t)-\mathbf{G}_{0}^{*} u_{x x}(\mathbf{x}, t)-\int_{0}^{\infty} \mathbf{G}^{\prime}(s) u_{x x}(\mathbf{x}, t-s) d s=0, & (\mathbf{x}, t) \in\left(0, l^{*}\right) \times \mathbb{R}^{+}, \\ u(\mathbf{x}, t)=u^{*}(\mathbf{x},-t), & (\mathbf{x}, t) \in\left(0, l^{*}\right) \times \mathbb{R}^{-} \\ u(0, t)=u\left(l^{*}, t\right)=0, & t \in \mathbb{R}^{+}\end{cases}
$$

has a unique periodic (hence not decaying) solution.

Proof. First, we notice that (4.4) is well defined. Indeed, because of (4.3) we have

$$
\mathbf{G}_{0}^{*}+\mathbf{G}_{c}^{\prime}\left(\omega^{*}\right)=\mathbf{G}_{\infty}^{*}-\int_{0}^{\infty} \mathbf{G}^{\prime}(s)\left(1-\cos \omega^{*} s\right) d s>0 .
$$

On account of $(4.5)$, past history $u^{*}$ belongs to $L^{\infty}\left(\mathbb{R}^{+}, H_{0}^{1}\left(0, l^{*}\right) \cap H^{2}\left(0, l^{*}\right)\right)$ and, by virtue of Remark 4 , it is trivially seen to belong to $\mathcal{U}^{*}$ for any $c \in \mathbb{R}$. Bearing in mind the statement of Theorem 1, there exists only one solution to (4.6) that agrees with the prescribed initial-history. A straightforward calculation shows that such a solution is given by

$$
u(\mathbf{x}, t)=c \sin \left(\frac{\pi \mathbf{x}}{l^{*}}\right)\left(\cos \omega^{*} t-\sin \omega^{*} t\right), \quad(\mathbf{x}, t) \in\left(0, l^{*}\right) \times \mathbb{R}^{+} .
$$

Since $\frac{\left(\omega^{*}\right)^{2}}{\mathbf{G}_{0}^{*}+\mathbf{G}_{c}^{\prime}\left(\omega^{*}\right)}$ turns out to be an eigenvalue of $-\Delta$ on $\left(0, l^{*}\right), u$ solves $(4.6)$ but it does not exhibit any decay in time.

For instance, let us consider the following three-parameter family of relaxation functions, exhibited first by Fabrizio and Morro in [17]:

$$
\mathbf{G}(t)=\mathbf{G}_{\infty}+\int_{t}^{\infty}\left\{s^{2}-\frac{\alpha-1}{\beta} s+\frac{\alpha^{2}}{8 \beta^{2}}\right\} \exp (-\beta s) d s
$$

where $\alpha \in \mathbb{R}$ and $\beta>0, \mathbf{G}_{x}>0$ in order to comply with $\mathbb{P}_{1}, \mathbb{P}_{2}, \mathbb{P}_{3}$, and $\mathbb{P}_{5}$. If $0<\alpha<8$, the associated Boltzmann function $\mathbf{G}^{\prime}$ meets $\mathbb{P}_{4}^{\prime}$, too. Actually, we have

$$
\mathbf{G}_{s}^{\prime}\left(\omega^{*}\right)=0 \text { for } \omega^{*}=\beta \sqrt{\frac{8-\alpha}{\alpha}}, \text { and } \mathbf{G}_{s}^{\prime}(\omega)<0 \quad \text { if } \omega \neq \omega^{*}
$$


Remarkably, relaxation functions (4.7) corresponding to $2-\sqrt{2}<\alpha<2+\sqrt{2}$ are positive and decreasing. In addition, both properties (a) and (b) of Remark 4 are satisfied by all functions of this family. In this case Theorem 6 applies, and relations (4.3)-(4.4) yield

$$
\begin{gathered}
\mathbf{G}_{\infty}^{*}>\frac{1}{16 \beta^{3}}(8-\alpha)(\alpha-6), \\
l^{*}=\pi \sqrt{\left[\mathbf{G}_{\infty}^{*}-\frac{(8-\alpha)(\alpha-6)}{16 \beta^{3}}\right] \frac{\alpha}{(8-\alpha) \beta^{2}}} .
\end{gathered}
$$

Thereby, for any given $l$ and $\mathbf{G}_{\infty}$ (both positive) it is always possible to determine $\alpha \in(0,8)$ and $\beta \in \mathbb{R}^{++}$so that system (4.6) has a unique periodic solution.

5. Some remarks on invertibility. Finally, we shall prove that $\mathbb{P}_{1}-\mathbb{P}_{4}$ allow the invertibility of the constitutive functional (4.1). The point of view taken here is close to that assumed in [18] and, especially, in [19]. The result we achieve, however, is somewhat different with regard to the function spaces involved. Actually, we show the well-posedness of the inversion problem from $L^{1}$, or $L^{\infty}$, into itself. To this end we recall a result by Benvenuti:

Lemma 2 ([20], Teorema F). Let $G^{\prime} \in L^{1}$ be a causal function, ${ }^{4}$ and let $\boldsymbol{T}$ be the memory functional defined by (4.1), namely

$$
\mathbf{T}(t)=\boldsymbol{T}(\mathbf{E}(\cdot))(t)=\mathbf{G}_{0} \mathbf{E}(t)+\int_{0}^{\infty} \mathbf{G}^{\prime}(s) \mathbf{E}(t-s) d s .
$$

Then, $\boldsymbol{T}$ is invertible on $L^{1}\left(\right.$ or $L^{\infty}$ ), i.e., there exists a causal function $\mathbf{J}^{\prime} \in L^{1}$ such that

$$
\mathbf{E}(t)=\boldsymbol{T}^{-1}(\mathbf{T}(\cdot))(t)=\mathbf{J}_{0} \mathbf{T}(t)+\int_{0}^{\infty} \mathbf{J}^{\prime}(s) \mathbf{T}(t-s) d s, \quad \mathbf{J}_{0}=\frac{1}{\mathbf{G}_{0}}
$$

if and only if $\left|\mathbf{G}_{0}+\widetilde{\mathbf{G}}^{\prime}(z)\right|>\delta$ on the complex half-plane $\operatorname{Re} z \geq 0$, where $\delta$ is a suitable positive constant and $\widetilde{\mathbf{G}}^{\prime}$ is the Laplace transform of $\mathbf{G}^{\prime}$, namely

$$
\widetilde{\mathbf{G}}^{\prime}(z)=\int_{0}^{\infty} \mathbf{G}^{\prime}(s) \exp (-z s) d s, \quad z \in \mathbb{C}, \operatorname{Re} z \geq 0 .
$$

Thereby, our goal is achieved by means of the following Theorem, which is a simpler version of a previous result for tensor-valued relaxation functions.

Theorem 7 ([21], Teorema 5). If the relaxation function $\mathbf{G}$ satisfies $\mathbb{P}_{1}-\mathbb{P}_{4}$, then there exists a positive constant $\delta$ such that

$$
\left|\mathbf{G}_{0}+\widetilde{\mathbf{G}}^{\prime}(z)\right|>\delta, \quad \forall z \in \mathbb{C}, \operatorname{Re} z \geq 0 .
$$

Proof. By Parseval's Lemma on Fourier transforms we have

$$
\int_{-\infty}^{\infty} f(s) g(s) \exp (-i \omega s) d s=\frac{1}{2 \pi} \int_{-\infty}^{\infty} \hat{f}(\tau) \hat{g}(\omega-\tau) d \tau
$$

\footnotetext{
${ }^{4} \mathrm{~A}$ causal function is a function defined on $\mathbb{R}$ and vanishing on $\mathbb{R}^{-}$.
} 
Letting $\alpha>0$, we choose $f$ and $g$ as follows:

$$
f(s)=\left\{\begin{array}{ll}
-\mathbf{G}^{\prime}(s), & s<0, \\
\mathbf{G}^{\prime}(s), & s \geq 0,
\end{array}, \quad g(s)= \begin{cases}0, & s<0 \\
\exp (-\alpha s), & s \geq 0\end{cases}\right.
$$

Because of $\mathbb{P}_{1}$ and $\mathbb{P}_{2}$ the expression (5.1) is well defined and yields

$$
\widetilde{\mathbf{G}}^{\prime}(\alpha+i \omega)=-\frac{i}{\pi} \int_{-\infty}^{\infty} \frac{\mathbf{G}_{s}^{\prime}(\tau)}{(\alpha+i \omega)-i \tau} d \tau=\frac{2}{\pi} \int_{-\infty}^{\infty} \frac{\tau \mathbf{G}_{s}^{\prime}(\tau)}{\alpha^{2}+\tau^{2}-\omega^{2}+2 i \alpha \omega} d \tau
$$

whence, splitting $\widetilde{\mathbf{G}}^{\prime}$ into its real and imaginary part for $\alpha>0$,

$$
\begin{aligned}
& \operatorname{Re} \widetilde{\mathbf{G}}^{\prime}(\alpha+i \omega)=\frac{2}{\pi} \int_{0}^{\infty} \frac{\tau\left(\alpha^{2}+\tau^{2}-\omega^{2}\right) \mathbf{G}_{s}^{\prime}(\tau)}{\left(\alpha^{2}+\tau^{2}-\omega^{2}\right)^{2}+4 \alpha^{2} \omega^{2}} d \tau \\
& \operatorname{Im} \widetilde{\mathbf{G}}^{\prime}(\alpha+i \omega)=-\frac{4 \alpha \omega}{\pi} \int_{0}^{\infty} \frac{\tau \mathbf{G}_{s}^{\prime}(\tau)}{\left(\alpha^{2}+\tau^{2}-\omega^{2}\right)^{2}+4 \alpha^{2} \omega^{2}} d \tau
\end{aligned}
$$

and, moreover,

$$
\operatorname{Re} \widetilde{\mathbf{G}}^{\prime}(i \omega)=\mathbf{G}_{c}^{\prime}(\omega), \quad \operatorname{Im} \widetilde{\mathbf{G}}^{\prime}(i \omega)=-\mathbf{G}_{s}^{\prime}(\omega) .
$$

On account of $\mathbb{P}_{4}$, from the latter expression and (5.3) we obtain

$$
\operatorname{Im} \widetilde{\mathbf{G}}^{\prime}(\alpha+i \omega) \neq 0, \quad \forall \omega \in \mathbb{R} \backslash\{0\}, \alpha \in \mathbb{R}^{+}
$$

since $\operatorname{Im} \widetilde{\mathbf{G}}^{\prime}(\alpha+i \omega)$ is an odd function with respect to $\omega$.

Now, bearing in mind (1.5), namely

$$
\mathbf{G}_{0}=\mathbf{G}_{\infty}-\frac{2}{\pi} \int_{0}^{\infty} \frac{\mathbf{G}_{s}^{\prime}(\tau)}{\tau} d \tau
$$

from (5.2) we obtain

$$
\begin{aligned}
\mathbf{G}_{0}+\operatorname{Re} \widetilde{\mathbf{G}}^{\prime}(\alpha) & =\mathbf{G}_{0}+\frac{2}{\pi} \int_{0}^{\infty} \frac{\tau \mathbf{G}_{s}^{\prime}(\tau)}{\left(\alpha^{2}+\tau^{2}\right)} d \tau \\
& =\mathbf{G}_{\infty}-\frac{2 \alpha^{2}}{\pi} \int_{0}^{\infty} \frac{\mathbf{G}_{s}^{\prime}(\tau)}{\tau\left(\alpha^{2}+\tau^{2}\right)} d \tau
\end{aligned}
$$

so that, on account of $\mathbb{P}_{3}$ and $\mathbb{P}_{4}$, it follows that

$$
\mathbf{G}_{0}+\operatorname{Re} \widetilde{\mathbf{G}}^{\prime}(\alpha+i \omega)>\mathbf{G}_{\infty}>0, \quad \omega=0, \forall \alpha \in \mathbb{R}^{+} .
$$

Trivially, inequalities (5.4), (5.5), and (1.5) yield

$$
\left|\mathbf{G}_{0}+\widetilde{G}^{\prime}(z)\right|>0, \quad \forall z \in \mathbb{C}, \operatorname{Re} z \geq 0,
$$

and the thesis easily follows by pointing out that conditions $\mathbb{P}_{2}-\mathbb{P}_{3}$ lead to

$$
\lim _{|z| \rightarrow+\infty, \operatorname{Re} z \geq 0}\left[\mathbf{G}_{0}+\widetilde{\mathbf{G}}^{\prime}(z)\right]=\mathbf{G}_{0}>0 .
$$

Acknowledgment. This work has been performed under the auspices of G.N.F.M.C.N.R. and partially supported by the Italian M.U.R.S.T. through the $40 \%$ Project "Problemi di evoluzione nei fluidi e nei solidi". 


\section{REFERENCES}

[1] B. D. Coleman and W. Noll, Foundations of linear viscoelasticity, Rev. Mod. Phys. 33, 239-249 (1961)

[2] M. J. Leitman and G. M. C. Fisher, The linear theory of viscoelasticity, in Encyclopedia of Physics, Vol. VIa/3, C. Truesdell ed., Springer-Verlag, Berlin, 1973

[3] W. A. Day, The Thermodynamics of Simple Materials with Fading Memory, Springer, Berlin, 1972

[4] M. Fabrizio and A. Morro, Mathematical Problems in Linear Viscoelasticity, SIAM, Philadelphia, 1992

[5] W. A. Day, Time-reversal and the symmetry of the relaxation function of a linear viscoelastic material, Arch. Rational Mech. Anal. 40, 155-159 (1971)

[6] M. Fabrizio and A. Morro, Viscoelastic relaxation function compatible with thermodynamics, J. Elasticity 19,63-75 (1988)

[7] M. Fabrizio, C. Giorgi, and A. Morro, Free energies and dissipation properties for systems with memory, Arch. Rational Mech. Anal. 125, 341-373 (1994)

[8] M. Fabrizio, Proprietà di stabilità per sistemi con memoria, Atti Acc. Peloritana dei Pericolanti, Classe I - Sc. Fis. Mat. Nat., 68, Suppl.n.1 (1990)

[9] G. Duvault and J. L. Lions, Inequalities in Mechanics and Physics, Springer, Berlin, 1976

[10] C. M. Dafermos, Asymptotic stability in viscoelasticity, Arch. Rational Mech. Anal. 37, 297-308 (1970)

[11] M. Fabrizio and B. Lazzari, On the existence and the asymptotic stability of solutions for linearly viscoelastic solids, Arch. Rational Mech. Anal. 116, 139-152 (1991)

[12] C. Baiocchi, Soluzioni ordinarie e generalizzate del problema di Cauchy per equazioni differenziali astratte non lineari del secondo ordine in spazi di Hilbert, Ricerche Mat. 16, 27-95 (1967)

[13] B. Lazzari and E. Vuk, Un teorema di esistenza e unicità per un problema dinamico in viscoelasticità lineare, Atti Sem. Mat. Fis. Univ. Modena 35, 309-317 (1987)

[14] M. Fabrizio, An existence and uniqueness theorem in quasi-static viscoelasticity, Quart. Appl. Math. 47, 1-8 (1989)

[15] C. Giorgi and B. Lazzari, Uniqueness and stability in linear viscoelasticity: some counterexamples, in Proceedings of the Vth International Meeting on Waves and Stability in Continuous Media, Sorrento, Italy, 9-14 October, 1989, ed. S. Rionero, World Scientific Publishing, River Edge, NJ, 1991 , pp. $146-153$

[16] M. E. Gurtin and I. Herrera, On dissipation inequalities and linear viscoelasticity, Quart. Appl. Math. 23, 235-245 (1965)

[17] M. Fabrizio and A. Morro, On uniqueness in linear viscoelasticity: a family of counterexamples, Quart. Appl. Math. 45, 263-268 (1987)

[18] M. Fabrizio, Sull'invertibilità dell'equazione costitutiva della viscoelasticità lineare, Rend. Mat. Acc. Naz. Lincei, (9) 3, 141-148 (1992)

[19] G. Gentili and A. Bozza, Inversion and quasi-static problems in linear viscoelasticity, Meccanica (to appear)

[20] P. Benvenuti, Sulla teoria ereditaria delle deformazioni lineari dinamiche, Rend. Mat. 24, 255-290 (1965)

[21] C. Giorgi, Alcune conseguenze delle restrizioni termodinamiche per mezzi viscoelastici lineari, Quad. Dip. Mat. Univ. Cattolica Brescia, 6/89 (internal report) 